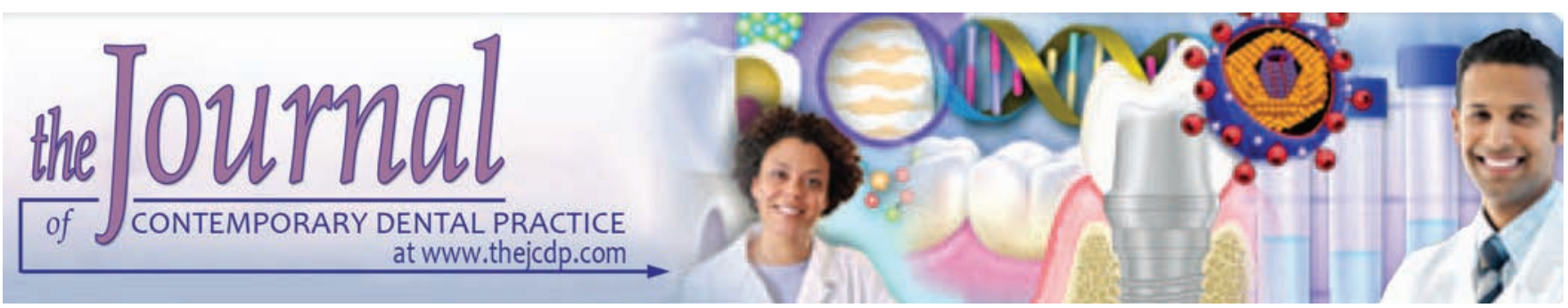

\title{
Evaluation of Antibacterial Effect and Dimensional Stability of Self-disinfecting Irreversible Hydrocolloid: An in vitro Study
}

${ }^{1}$ Veena B Benakatti, ${ }^{2}$ Abhijit P Patil, ${ }^{3}$ Jayashee Sajjanar, ${ }^{4}$ Supriya S Shetye, ${ }^{5}$ Ulhas N Amasi, ${ }^{6}$ Raghunath Patil

\begin{abstract}
Aim: This study evaluated the antibacterial activity and dimensional stability of irreversible hydrocolloids mixed with different concentrations of chlorhexidine gluconate instead of water.

Materials and methods: Experimental specimens (45 specimens) were prepared and allocated into three groups of 15 each. Group I: Impression material mixed with distilled water served as control. Groups II and III were prepared with 0.12 and $0.2 \%$ chlorhexidine gluconate solution, respectively. Specimens in each group were subjected to tests for dimensional stability. For antimicrobial activity, 30 specimens were prepared and allocated into three groups of 10 each named as group I (control), group II ( $0.12 \%$ chlorhexidine gluconate), and group III $(0.2 \%$ chlorhexidine gluconate) similar to specimens for dimensional stability. Statistical analysis was performed using a one-way analysis of variance (ANOVA) and Tukey test.
\end{abstract}

Results: Zones of inhibition were observed around test specimens, but not around control specimens; there was a significant intergroup difference in the diameters of the inhibition zones. In the test for dimensional stability, no significant differences were detected among groups, and the accuracy was clinically acceptable.

Conclusion: Irreversible hydrocolloid impression material mixed with chlorhexidine exhibits varying degrees of antibacterial activity without influencing the dimensional stability of set material.

Clinical significance: Many contagious diseases can be prevented by practical control of infection in the dental office. Chlorhexidine gluconate, as a mixing liquid, ensures disinfection of impression, and this method of disinfection is more convenient and avoids extra effort as in other disinfection techniques.

\footnotetext{
1,2,5,6 Department of Prosthodontics, KLE VK Institute of Dental Sciences, KLE University, Belagavi, Karnataka, India

${ }^{3}$ Department of Prosthodontics, Swargiya Dadasaheb Kalmegh Smruti Dental College, Nagpur, Maharashtra, India

${ }^{4}$ Private Dental Practice, Goa, India

Corresponding Author: Veena B Benakatti, Department of Prosthodontics, KLE VK Institute of Dental Sciences, KLE University, Belagavi, Karnataka, India, Phone: +919481357779 e-mail: veenak20021@rediffmail.com
}

Keywords: Antibacterial activity, Chlorhexidine, Dimensional stability, Disinfection, Irreversible hydrocolloid.

How to cite this article: Benakatti VB, Patil AP, Sajjanar J, Shetye SS, Amasi UN, Patil R. Evaluation of Antibacterial Effect and Dimensional Stability of Self-disinfecting Irreversible Hydrocolloid: An in vitro Study. J Contemp Dent Pract 2017;18(10):887-892.

Source of support: Nil

Conflict of interest: None

\section{INTRODUCTION}

As a health professional, it is the responsibility of the dental surgeon to prevent disease and treat when disease sets in. The American Dental Association (ADA) has recommended use of gloves, caps, masks, eyeglasses, and sterilization of dental materials for control of infection in dental offices and laboratories. Impressions along with trays are also an important source of cross-contamination, which should be considered in infection control. ${ }^{1}$

In dental clinics, dental impressions are easily contaminated with saliva and blood from the oral cavity. ${ }^{2}$ These contaminated impressions, particularly hydrocolloid impressions, pose notable risks to dental practitioners, patients, and laboratory personnel., ${ }^{3,4}$ Due to hydrophilic nature, irreversible hydrocolloids retain the highest number of bacteria. ${ }^{5}$ Leung and Schonfeld ${ }^{6}$ reported that even stone casts are potential sources of cross-infection. Prosthetic work can expose professionals and technicians to contagious diseases, such as acquired immunodeficiency syndrome, hepatitis, and tuberculosis. Hence, additional infection control procedures, such as washing and disinfecting impressions should be followed during the manipulation and handling of dental impressions and dentures. ${ }^{1}$

Many studies have evaluated the effect of various disinfectants and methods of disinfecting impression 
materials, but the results of those studies varied widely. Disinfectant should ideally be an effective antimicrobial agent, yet cause no adverse effects on properties of impression material and the resultant gypsum cast. ${ }^{2}$

Many methods have been proposed for disinfection of irreversible hydrocolloid impression material. Among these, spray and immersion are the most common methods used in clinical practice. However, these methods present several disadvantages. Disinfection by immersion or spraying is quite effective in reducing cross-infection, but compliance by dental professionals has been uneven. The reasons for this include:

- Disinfection involves an extra effort or action.

- Loss of surface detail and dimensional accuracy of the impression by spraying or immersing impression material with disinfectants.

- Inhalation of disinfectants used for spray and immersion techniques may present health risks to the dental team.

- Toxic disinfectants cause corrosion of metallic trays or dislodgement of the impression from the tray. ${ }^{7}$

Due to these difficulties, self-disinfecting irreversible hydrocolloid impression materials evolved as those are impregnated with disinfectants. This technique reduced the overall quantity of bacteria on the impression material, without affecting dimensional stability than spray and immersion techniques, and saved disinfection time.?

However, disinfectant was impregnated into the powder of impression material for most of the selfdisinfecting irreversible hydrocolloid impression materials and few attempts have been made to add disinfectants into the mixing liquid. Hence, in the present study, various concentrations of chlorhexidine gluconate solution were used to mix the irreversible hydrocolloid impression material powder, and the antibacterial effect and dimensional accuracy of impression material were evaluated.

The purpose of the study was to determine the following:

- Whether in vitro antibacterial activity against representative pathogenic microbes could be obtained after mixing chlorhexidine gluconate with irreversible hydrocolloid impression material

- The effects of chlorhexidine solution on the dimensional accuracy of irreversible hydrocolloid impression material

- The concentration of chlorhexidine recommended for producing the self-disinfecting impression material

\section{MATERIALS AND METHODS}

\section{Impression Materials and Gypsum Products}

Irreversible hydrocolloid impression material generally used in prosthodontic and orthodontic practice was selected for the study. The commercial alginate Tropicalgin (Zhermack) and a range of gypsum products commonly used in clinical practice were used in the study. Impression material and gypsum products were manipulated according to manufacturer's instructions.

\section{Disinfection Procedures}

The disinfecting solution used was chlorhexidine gluconate solution in two concentrations, namely, 0.12 and $0.2 \%$. Chlorhexidine was used in its pure form with no additives and preservatives (ICPA health products Ltd., Ahmedabad, India). Distilled water was used as a control. Chlorhexidine was used as mixing liquid for irreversible hydrocolloid.

\section{Measurement of Dimensional Accuracy}

A master die according to the ADA specification (specification no. 18$)^{8}$ was used in the study as shown in Figure 1. A stainless steel block was machined into a round base and a platform. Two points, namely $\mathrm{x}$ and $\mathrm{x}^{\prime}$, were identified on the master die, which were formed at the intersection of the $25 \mu \mathrm{m}$ (width) vertical line and the two horizontal lines $d_{1}$ and $d_{2}$. Then, the distance between points $x$ and $x^{\prime}$ was measured using an electronic caliper to evaluate the dimensional stability. As shown in Figure 1, custom tray for this steel die was fabricated. A wax spacer was positioned over the entire master die, resembling an inverted loaf to provide uniform spacing ( $2 \mathrm{~mm}$ ) and consistent seating against the die base for the acrylic resin trays. Custom trays were fabricated over the die. Uniformly spaced perforations were placed in the trays with a round bur to retain the irreversible hydrocolloid without an adhesive. Irreversible hydrocolloid impression material was mixed according to manufacturer's instructions. Chlorhexidine gluconate solution was used as mixing liquid for test groups and autoclaved

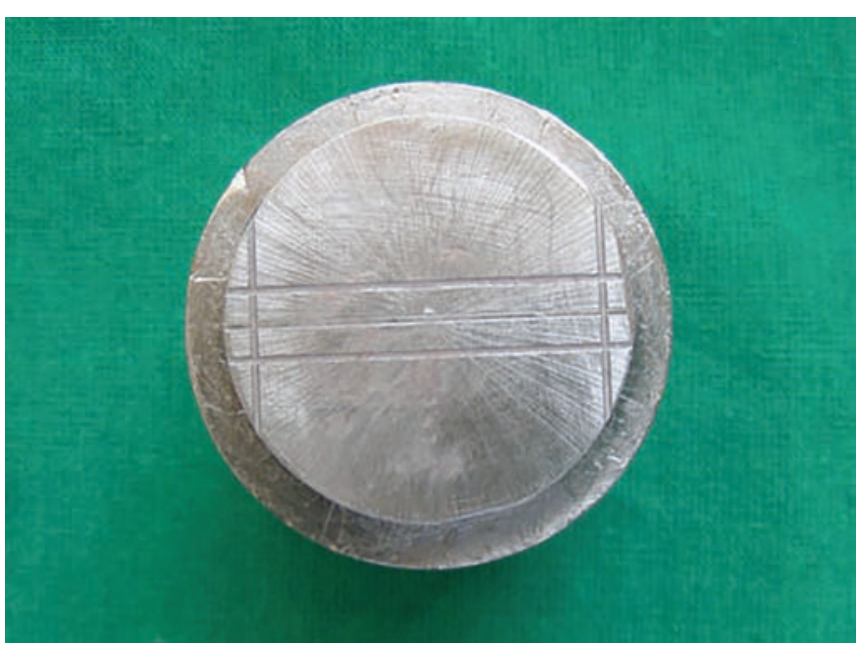

Fig. 1: ADA specification no. 18 master die 
distilled water for control group. Impressions of the steel die were made with the custom tray, and allowed to set for 6 minutes at room temperature with uniform pressure on the die. Impressions were poured immediately in die stone, and stone casts were recovered. Die stone was mixed according to manufacturer's instructions and mechanically spatulated with a stirring instrument. The stone casts were allowed to set for 2 hours before separation and were dried at room temperature for at least 24 hours before being measured. Measurements were made by single operator using an electronic digital caliper between two designated points on the specimen. Mean dimensions of the stone cast (control or after disinfection), which were obtained by calculating the mean of three measurements for the stone cast, were compared with a mean of three readings of master die. Results were then subjected to statistical analysis.

The dimensional stability was calculated using the following formula ${ }^{2}$ :

$$
\left(L-L^{\prime} / L\right) \times 100
$$

where $L$ represents the dimensions of the master die which were obtained by calculating the mean of three measurements; L' represents the mean dimension of the stone cast (control or after disinfection) which were obtained by calculating the mean of three measurements for the stone cast.

\section{Preparation of Test and Control Specimens}

Specimens made from irreversible hydrocolloid impression material were prepared in accordance with the specific requirements in different tests. The specimens were divided into three groups in each test: Group I specimens prepared by mixing irreversible hydrocolloid with autoclaved distilled water which served as control, group II specimens were mixed with $0.12 \%$ chlorhexidine solution, and group III specimens were mixed with $0.2 \%$ chlorhexidine solution.

\section{Measurement of Antibacterial Effect}

The agar well technique was used to assess the antibacterial activity of the specimens. Irreversible hydrocolloid impression material was mixed according to the powder/liquid ratio recommended by the manufacturer. Then impression disks, $6 \mathrm{~mm}$ in diameter and $1 \mathrm{~mm}$ in thickness, were prepared. These impression disks were embedded in nutrient agar plates previously inoculated with the appropriate microorganisms under sterile conditions. On each agar plate, two impression disks were embedded from each of the two test groups (group $0.12 \%$, group $0.2 \%$ ). The control specimen (autoclaved distilled water) was placed in each plate (Fig. 2). Finally, all plates were incubated in the appropriate aerobic environment for 24 hours at $37^{\circ} \mathrm{C}$.

After incubation, the clear zones or inhibitory areas appeared around the specimens (Fig. 3). Inhibitory zones were measured with an antibiotic zone scale to evaluate the antibacterial effect (Fig. 4). The following microorganisms were used: American Type Culture Collection (ATCC) Staphylococcus aureus ATCC 29213 and S. aureus ATCC 33591.

\section{RESULTS}

The means and standard deviations of the dimensional changes are presented in Table 1. Control specimens showed greater dimensional accuracy in comparison with test groups. However, there was no significant difference in dimensional accuracy between impression materials mixed with different concentrations of disinfectant solution. However, the differences in dimensional accuracy of three groups were statistically nonsignificant

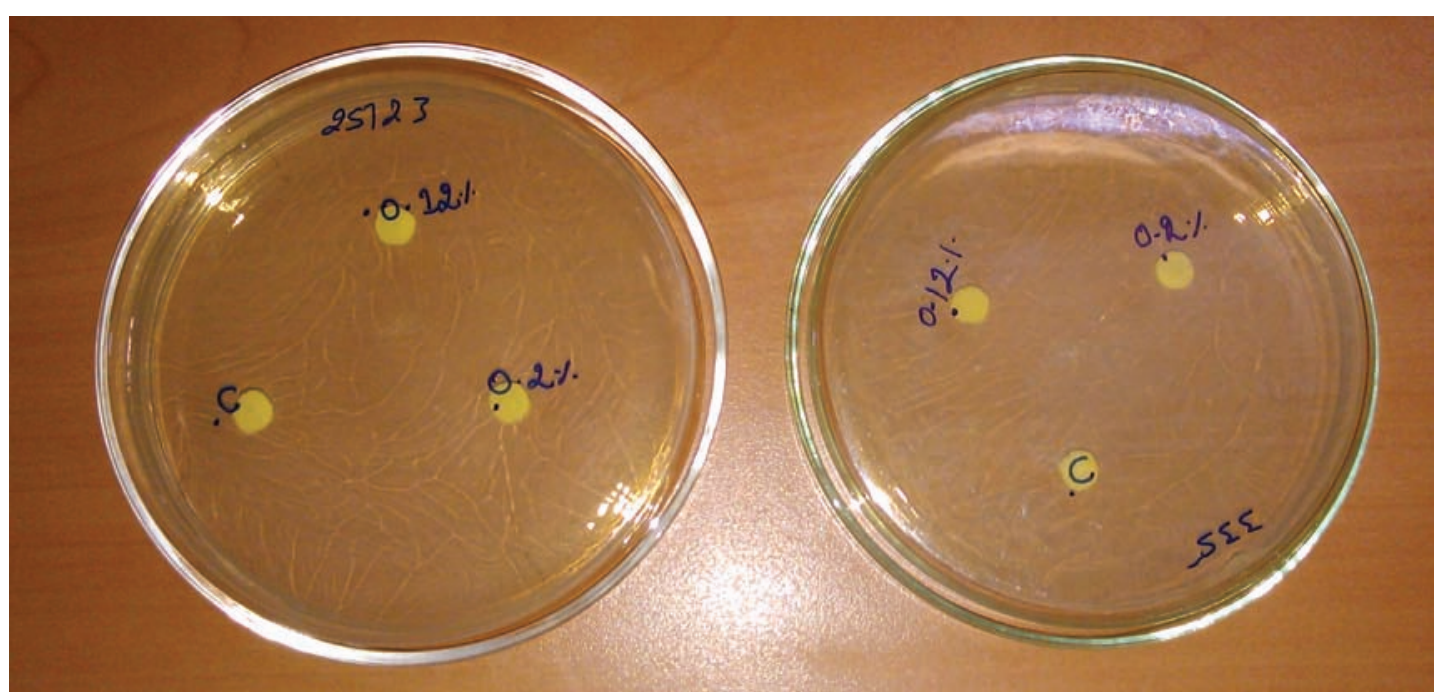

Fig. 2: Specimens embedded in media inoculated with microorganisms 


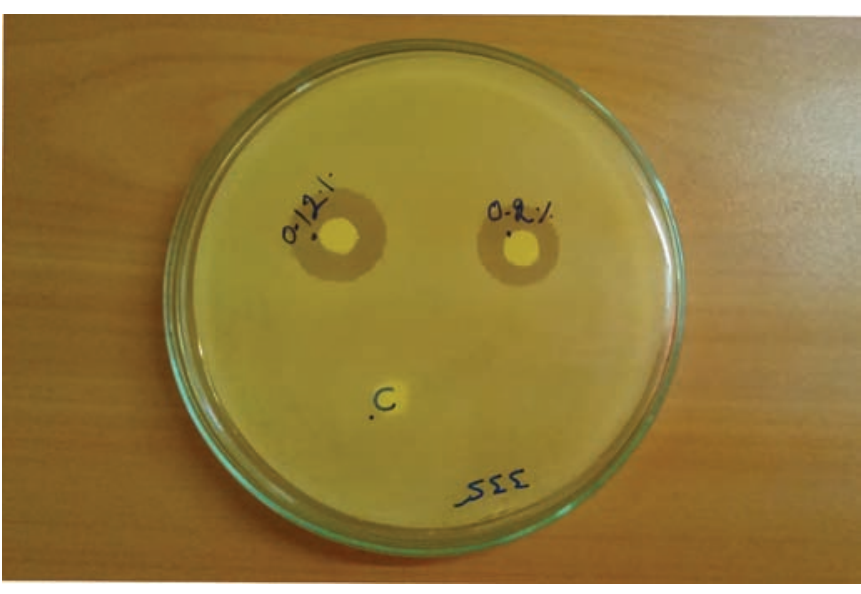

Fig. 3: Inhibitory zones after 24 hour incubation period

Table 1: Mean, SD, SE, and coefficient of variation of dimensional change scores in three study groups

\begin{tabular}{llll}
\hline Groups & Mean \pm SD & SE & $\begin{array}{l}\text { \% of dimensional } \\
\text { change }\end{array}$ \\
\hline Control distilled water & $24.96 \pm 0.03$ & 0.01 & 0.15 \\
$0.12 \%$ chlorhexidine & $24.64 \pm 0.35$ & 0.11 & 1.42 \\
$0.2 \%$ chlorhexidine & $24.66 \pm 0.34$ & 0.10 & 1.37 \\
\hline
\end{tabular}

SE: Standard error, SD: Standard deviation

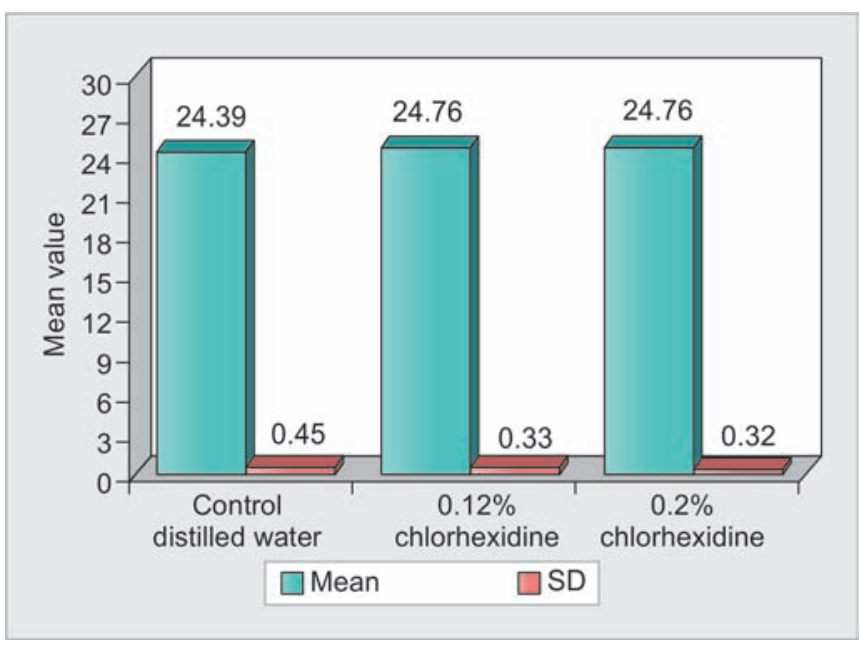

Graph 1: Comparison of three study groups with mean dimensional change scores

$(\mathrm{p}<0.05)$ (Graph 1). Table 2 summarizes comparison of the study groups with mean dimensional change scores by one-way ANOVA with no significant differences $(p<0.05)$ among groups in relation to each other.

Well-defined zones of inhibition became apparent after incubation period, and consistent measuring of inhibitory fields was done. Mean diameters of inhibited zones for each microorganism are presented in Table 3. The results demonstrated zones of inhibition around the test specimens on all plates. No zones of inhibited growth were observed around the control wells on agar plates. One-way ANOVA and Tukey test revealed that the inhibition zones tested became significantly larger for each

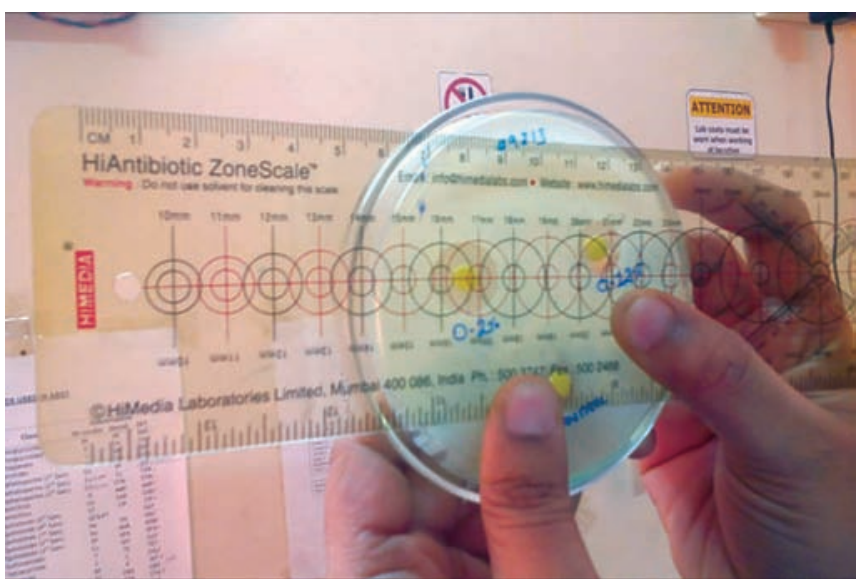

Fig. 4: Measurement of inhibitory zones

Table 2: Comparison of three study groups with mean dimensional change scores by one-way ANOVA

\begin{tabular}{llllll}
\hline $\begin{array}{l}\text { Source of } \\
\text { variation }\end{array}$ & $\begin{array}{l}\text { Degrees of } \\
\text { freedom }\end{array}$ & $\begin{array}{l}\text { Sum of } \\
\text { squares }\end{array}$ & $\begin{array}{l}\text { Mean sum } \\
\text { of squares }\end{array}$ & f-value & $p$-value \\
\hline $\begin{array}{l}\text { Between } \\
\text { groups }\end{array}$ & 2 & 1.29 & 0.6447 & 8.0952 & $0.0008^{*}$ \\
$\begin{array}{l}\text { Within } \\
\text { groups }\end{array}$ & 57 & 4.54 & 0.0796 & & \\
\hline Total & 59 & 5.83 & & & \\
\hline${ }^{*} \mathrm{p}<0.05$ & & & & & \\
\end{tabular}

Table 3: Mean diameter of inhibition zone $(\mathrm{mm})$ for each bacterial species and group

\begin{tabular}{llll}
\hline Species & $0.12 \%$ & $0.2 \%$ & Control \\
\hline S. aureus ATCC 33591 & 14 & 15 & 0 \\
S. aureus ATCC 29213 & 19 & 20 & 0 \\
\hline
\end{tabular}

microorganism when the concentrations of chlorhexidine solution were raised from 0.12 to $0.2 \%$.

\section{DISCUSSION}

Decontamination of impression materials is an essential stage in controlling cross-infection in dentistry. ${ }^{9}$ Use of disinfectant-supplemented irreversible hydrocolloid impression materials provides greater efficiency and effectiveness compared with the other disinfection techniques. ${ }^{7}$

Oral microorganisms easily get incorporated in impression materials during setting. Immersing or spraying rinsed impressions provide only surface disinfection. However, the self-disinfecting impression is uniformly disinfected throughout the material and not just on the surface. Impression material takes up oral fluids and microbes while setting, which introduces microorganisms within the material. Therefore, disinfectant-supplemented alginates could eliminate most of the microbes that were incorporated into the set impression materials. ${ }^{7}$ 
Chlorhexidine is a broad-spectrum disinfectant, i.e., an effective antiplaque and antigingivitis agent. Chlorhexidine has been studied in several mouthrinse formulations and is proved to be safe and effective. ${ }^{7}$ Most commonly used concentrations of chlorhexidine solutions as mouth rinse are 0.12 and $0.2 \%$; these concentrations are proved to be effective against most of the microbes. To be indicated as a substitute for water, the solution used for the mix should be biocompatible and not affect either the physical or mechanical properties of the irreversible hydrocolloid. Chlorhexidine gluconate solution was selected for this research because it fulfills those requirements. Chlorhexidine was used in its pure form without any additives to eliminate effects of other ingredients on properties impression material. Thickness of samples to test for antibacterial activity was kept uniform, i.e., $2 \mathrm{~mm}$ to achieve consistent results.

Disinfection method used in this study is shown to be effective against $S$. aureus, which is in accordance with a study conducted by Casemiro et al ${ }^{10}$; they evaluated in vitro antimicrobial activity of irreversible hydrocolloids prepared with a $0.2 \%$ chlorhexidine gluconate solution against oral microbiota. They found that chlorhexidine was effective against $S$ aureus.

Staphylococcus aureus is found to be sensitive to chlorhexidine, which is in agreement with the study by Wang ${ }^{7}$ and Zahraa Alwahab. ${ }^{11}$ Positively charged chlorhexidine molecule interacts with negatively charged bacterial cell wall and permits agent to penetrate into cytoplasm leading to potassium leakage and death of microbe. ${ }^{11}$

It was expected that exposure of impression materials to disinfectants would adversely affect dimensional accuracy. ${ }^{9}$ However, results of this study show that the dimensional changes were insignificant when chlorhexidine was used as mixing liquid. Specimens made with distilled water were more dimensionally accurate in comparison to specimens made with 0.12 and $0.2 \%$ chlorhexidine solution.

The mean percentage deviation of measurements recorded for gypsum product produced comparable results. There was no significant difference in the overall dimensional accuracy between casts made from impression materials mixed with different concentrations of chlorhexidine. This is in agreement with study conducted by Taylor et al. ${ }^{9}$

The result of the current study is also in agreement with study conducted by Wang et al. ${ }^{7}$ The threedimensional accuracy of the irreversible hydrocolloid was not influenced, even if chlorhexidine solution served as the mixing liquid.

Acceptable methods for measuring dimensional accuracy of casts include measuring microscopes, micrometers, dial gauges, and calipers. ${ }^{9}$ Digital caliper was used in the study with good reproducibility between repeat readings of each measurement.
Ramer et al ${ }^{12}$ conducted a study to evaluate the accuracy of alginate impressions made with water to which iodine or chlorhexidine was added. They found that accurate casts can be made when either of the disinfectant is incorporated into alginate impression material. This is in agreement with results of this study.

Amalan et $\mathrm{al}^{13}$ evaluated properties of irreversible hydrocolloid impression materials mixed with disinfectant liquids. Impression material was mixed with 0.1 and $0.2 \%$ chlorhexidine, which is similar to the method employed in this study. They concluded this method of disinfection ensures disinfection of impressions without significantly altering its properties.

Flanagan et $\mathrm{l}^{14}$ evaluated antimicrobial activities of dental impression material. They found that alginate with chlorhexidine killed all the Gram-negative bacilli and majority of Gram-positive cocci and yeast. Chlorhexidine is an effective antimicrobial agent and ensures disinfection of impression.

Rosen and Touyz ${ }^{15}$ studied changes in working time and accuracy of alginate impression material when mixed with disinfectant solutions rather than water. Results indicate that when chlorhexidine is used as a substitute for water, it does not modify working time beyond acceptable limits and has no significant effect on dimensional accuracy of the material.

There was a deviation between master model and test specimens. This level of deviation would have greater significance if impressions were to be used to construct fixed prosthesis. Irreversible hydrocolloid impression materials are usually used in prosthetic and orthodontics purposes where the level of accuracy is less critical. ${ }^{9}$ Hence, chlorhexidine can be safely used as mixing liquid for irreversible hydrocolloid impression material.

\section{CONCLUSION}

1. Chlorhexidine self-disinfecting irreversible hydrocolloid impression material exhibits varying degrees of in vitro antibacterial activity. This disinfection method was effective in the elimination of $S$. aureus.

2. Based on the findings of this study, chlorhexidine can be used as mixing liquid for irreversible hydrocolloid, without influencing the dimensional stability of set material.

3. Based on the findings of this study, $0.2 \%$ is the recommended concentration for chlorhexidine solution to produce the self-disinfecting impression material.

\section{REFERENCES}

1. Adabo GL, Zanarotti E, Fonseca RG, Cruz CA. Effect of disinfectant agents on dimensional stability of elastomeric impression materials. J Prosthet Dent 1999 May;81(5): 621-624. 
2. Amina WM, Al-Alib MH, Al Tarawnehc SK, Tahad ST, Salehe MW, Ereifijf N. The effects of disinfectants on dimensional accuracy and surface quality of impression materials and gypsum casts. J Clin Med Res 2009 Jun;1(2):81-89.

3. Kugel G, Perry RD, Ferrari M, Lalicata P. Disinfection and communication practices: a survey of U.S. dental laboratories. J Am Dent Assoc 2000 Jun; 131(6):786-792.

4. Samaranayake LP, Hunjan M, Jennings KJ. Carriage of oral flora on irreversible hydrocolloid and elastomeric impression materials. J Prosthet Dent 1991 Feb; 65(2):244-249.

5. Samra RK, Bhide SV. Efficacy of different disinfectant systems on alginate and addition silicone impression materials of Indian and international origin: a comparative evaluation. J Indian Prosthodont Soc 2010 Sep;10(3):182-189.

6. Leung RL, Schonfeld SE. Gypsum casts as a potential source of microbial cross-contamination. J Prosthet Dent 1983 Feb;49(2):210-211.

7. Wang J, Wan Q, Chao Y, Chen Y. A self-disinfecting irreversible hydrocolloid impression material mixed with chlorhexidine solution. Angle Orthod 2007 Sep; 77(5):894-900.

8. American National Standards Institute. ADA Specification No. 18 for Alginate Impression Material. Revised ANSI/ ADA Specification No. 18-1992 Council on Dental Materials, Instruments and Equipment, Washington, DC.
9. Taylor R, Wright PS, Maryan C. Disinfection procedures: their effect on dimensional accuracy and surface equality of irreversible hydrocolloid impression materials and gypsum casts. Dent Mater 2002 Mar; 18(2):103-110.

10. Casemiro L, Souza FC, Panzeri $\mathrm{H}$, Martins $\mathrm{CH}$, Ito IY. In vitro antimicrobial activity of irreversible hydrocolloid impressions against 12 oral microorganisms. Braz Oral Res 2007 Oct-Dec;21(4):323-329.

11. Alwahab Z. Comparison of antimicrobial activities and compressive strength of alginate impression materials following disinfection procedure. J Contemp Dent Pract 2012 Jul 1; 13(4):431-435.

12. Ramer MS, Gerhardt DE, McNally K. Accuracy of irreversible hydrocolloid impression material mixed with disinfectant solutions. J Prosthodont 1993 Sep; 2(3):156-158.

13. Amalan A, Ginjupalli K, Upadhya N. Evaluation of properties of irreversible hydrocolloid impression materials mixed with disinfectant liquids. Dent Res J (Isfahan) 2013 Jan; 10(1):65-73

14. Flanagan DA, Palenik CJ, Setcos JC, Miller CH. Antimicrobial activities of dental impression materials. Dent Mater 1998 Nov; 14(6):399-404.

15. Rosen M, Touyz LZ. Influence of mixing disinfectant solutions into alginate on working time and accuracy. J Dent 1991 Jun; 19(3):186-188. 